Research Article

\title{
Strong Convergence on the Aggregate Constraint-Shifting Homotopy Method for Solving General Nonconvex Programming
}

\author{
Zhichuan Zhu $\mathbb{D}^{1}$ and Yeong-Cheng Liou $\mathbb{D}^{2,3}$ \\ ${ }^{1}$ School of Economics, Liaoning University, Shenyang, Liaoning 110136, China \\ ${ }^{2}$ Department of Healthcare Administration and Medical Informatics, and Research Center of Nonlinear \\ Analysis and Optimization, Kaohsiung Medical University, Kaohsiung 807, Taiwan \\ ${ }^{3}$ Department of Medical Research, Kaohsiung Medical University Hospital, Kaohsiung 807, Taiwan \\ Correspondence should be addressed to Yeong-Cheng Liou; simplex_liou@hotmail.com
}

Received 16 September 2020; Revised 20 November 2020; Accepted 22 November 2020; Published 15 December 2020

Academic Editor: Ching-Feng Wen

Copyright ( 2020 Zhichuan Zhu and Yeong-Cheng Liou. This is an open access article distributed under the Creative Commons Attribution License, which permits unrestricted use, distribution, and reproduction in any medium, provided the original work is properly cited.

In the paper, the aggregate constraint-shifting homotopy method for solving general nonconvex nonlinear programming is considered. The aggregation is only about inequality constraint functions. Without any cone condition for the constraint functions, the existence and convergence of the globally convergent solution to the K-K-T system are obtained for both feasible and infeasible starting points under much weaker conditions.

\section{Introduction}

Throughout, let $R^{n}, R_{+}^{n}$, and $R_{++}^{n}$ denote the $n$-dimensional Euclidean space, nonnegative orthant, and positive orthant of $R^{n}$, respectively. In the paper, the following general nonconvex nonlinear programming will be considered:

$$
\begin{array}{ll}
\min & f(x) \\
\text { s.t. } & g_{i}(x) \leq 0, i=1,2, \ldots, m \\
& h_{j}(x)=0, j=1,2, \ldots, l,
\end{array}
$$

where $x \in R^{n}$ and $f(x): R^{n} \longrightarrow R, g_{i}(x): R^{n} \longrightarrow R^{m}$, and $h_{j}(x): R^{n} \longrightarrow R^{l}$ are three continuously differentiable functions. Denote $\Omega=\left\{x \mid g_{i}(x) \leq 0, i=1,2, \ldots, m, h_{j}(x)=\right.$ $0, j=1,2, \ldots, l\}, \Omega^{0}=\left\{x \mid g_{i}(x)<0, i=1,2, \ldots, m, h_{j}(x)=\right.$ $0, j=1,2, \ldots, l\}$, and $I(x)=\left\{x \mid g_{i}(x)=0, i=1,2, \ldots, m\right\}$.

It is well known that the solution of the optimization problem can be obtained through solving the K-K-T system of the convex nonlinear problem, but for the nonconvex nonlinear problem, we can only obtain the solution to the $\mathrm{K}-\mathrm{K}-\mathrm{T}$ system of problem (1).
Homotopy method has been paid much attention as an important globally convergent computational method in finding solutions to various nonlinear problems since it was introduced and studied by Kellogg et al. [1], Smale [2], and Chow et al. [3]. However, the original homotopy is only single homotopy and needs much strong assumptions when solving nonlinear problems. In the 1990s, a combined homotopy interior point (CHIP) method was firstly proposed for solving nonconvex programming under the normal cone condition by Feng and Yu in [4]. From then on, various CHIP methods, as an efficiently implementable algorithm, were widely used and newly constructed for solving general nonconvex programming, fixed point problems, complementarity problems, variational inequality, and so on, see, e.g., [5-20].

In 2001, for reducing the dimension of the systems arising in the numerically tracing process and weakening convergent conditions, Yu et al. [21] proposed an aggregate constraint homotopy method (ACH method) for nonconvex programming by using the so-called aggregate function of the constraints. In 2018, a new ACH method for nonlinear programming problems with inequality and equality 
constraints was presented in [22]. However, the $\mathrm{ACH}$ method still belongs to CHIP since it requires the initial point which was also in the original feasible set. In 2006, to avoid the disadvantage of CHIP must choose the initial point in the feasible set, a constraint-shifting combined homotopy infeasible interior-point method in which the initial point can be chosen in both feasible and infeasible sets for solving nonlinear programming with only inequality constraints was proposed by $\mathrm{Yu}$ and Shang in $[23,24]$. In 2012, to extend the constraint-shifting combined homotopy method to solve the general nonlinear programming, another new combined homotopy infeasible interior-point method for solving nonconvex programming with both inequality and equality constraints was proposed in [25], in which only inequality constraints need to satisfy the normal cone condition. From then on, more constraint-shifting homotopy equations were constructed and extended for solving nonlinear programming, principal-agent problem, fixed point problem, and so on, see, e.g., [26-30]. However, these combined homotopy methods usually required some cone conditions for proving the strong convergence of the existence of the smooth homotopy pathway.

By the enlightenment of the above references, without any cone condition, an aggregate constraint combined homotopy infeasible interior-point method for solving nonconvex nonlinear programming with both inequality and equality constraints is constructed, and the global convergence under much weaker conditions is obtained in the paper.

The remainder of this paper is organized as follows. In Section 2, the homotopy equation is constructed, and some lemmas from differential topology are introduced. In Section 3 , the main results will be presented, and the existence and convergence of a smooth path from any given point in the infeasible set to the solution of K-K-T systems are proved. In Section 4 , the numerical algorithm is presented.

\section{Preliminaries}

The following assumptions will be used:

(A1) $\Omega$ is a bounded and connected set, $\Omega^{0} \neq \phi$.

(A2) $\forall x \in \Omega$, matrix $\left\{\nabla g_{i}(x)_{i \in I(x)}, \nabla h_{j}(x)\right\}$ is positive linearly independent at $x$, i.e.,

$$
\sum_{i \in I(x)} \alpha_{i} \nabla g_{i}(x)+\sum_{j=1}^{m} \beta_{j} \nabla h_{j}(x)=0, \quad \alpha_{i} \in R_{+}, \beta_{j} \in R \Longrightarrow \alpha_{i}=\beta_{j}=0 .
$$

By [21], the aggregate function $g(x, \mu)=\mu \ln \left[\sum_{i=1}^{m}\right.$ $\left.\exp \left(g_{i}(x) / \mu\right)\right]$, we have

$$
\begin{aligned}
(I) \nabla_{x} g(x, \mu) & =\sum_{i=1}^{m} \tau_{i}(x, \mu) \nabla g_{i}(x), \tau_{i}(x, \mu) \\
& \triangleq \frac{\exp \left(g_{i}(x) / \mu\right)}{\sum_{j=1}^{m} \exp \left(g_{j}(x) / \mu\right)}, \\
(I I) g(x) & \leq g(x, \mu) \leq g(x)+\mu \ln m .
\end{aligned}
$$

We construct the following shifted aggregate constraint function only with inequality constraint functions:

$$
\tilde{g}(x, \mu)=\mu \phi(x)+(\theta-\mu) g(x, \mu),
$$

where $\theta \in(0,1)$ is a parameter and $\phi(x)$ are convex and three continuously differentiable functions. Therefore, we have

$$
\widetilde{g}(x, \theta)=\theta \phi(x)
$$

and

$\lim _{\mu \longrightarrow 0^{+}} \tilde{g}(x, \mu)=\lim _{\mu \longrightarrow 0^{+}}[\mu \phi(x)+(\theta-\mu) g(x, \mu)]=\theta g(x, \mu)$.

Obviously, $\tilde{g}(x, \mu)$ are also three continuously differentiable functions; let $\Omega_{\mu}=\{x \mid \widetilde{g}(x, \mu) \leq 0\}, \quad \Omega_{\mu}^{0}=\{x \mid \tilde{g}$ $(x, \mu)<0\}, \partial \Omega_{\mu}=\Omega_{\mu} / \Omega_{\mu}^{0}$, and $\Omega(0)=\Omega$.

Lemma 1 (see [21]). If assumptions (A1) and (A2) hold, then there exists $\theta_{1}>0$, for $\forall \mu \in\left(0, \theta_{1}\right)$, and

$$
\nabla_{x} \tilde{g}(x, \mu) \neq 0, \quad \forall x \in \partial \Omega(\mu) .
$$

Lemma 2 (see [27]). If assumptions (A1) and (A2) and hold, there exists $\theta_{2}>0$, for $\forall \mu \in\left(0, \theta_{2}\right)$, and we have $\Omega_{\mu}$ which is a bounded and connected set, and $\Omega_{\mu}^{0}$ is nonempty.

Lemma 3. If assumptions (A1) and (A2) hold, there exists $\theta_{3}>0$, for any given feasible point $x$, and $\forall \mu \in\left(0, \theta_{3}\right]$, matrix $\left\{\left(\nabla_{x} \tilde{g}(x, \mu), \nabla h_{j}(x)\right\}\right.$ is positive linearly independent.

Proof. Proved by contradiction. For $\forall \mu_{k} \in\left(0, \theta_{3}\right]$ and any feasible point $x^{k}$, there exists $\alpha^{k} \geq 0$ and $\beta_{j}^{k} \in R$ belonging to real part, which are simultaneously not equal to zeros, such that

$$
\alpha^{k} \nabla_{x} \widetilde{g}\left(x^{k}, \mu_{k}\right)+\sum_{j=1}^{m} \beta_{j}^{k} \nabla h_{j}\left(x^{k}\right)=0 .
$$

Let $\beta^{k}=\max _{1 \leq j \leq l}\left\{\left|\beta_{j}^{k}\right|\right\}$ and $\xi^{k}=\max \left\{\alpha^{k}, \beta^{k}\right\}$; divide both sides of (8) by $\xi^{k}$, i.e., 


$$
\frac{\alpha^{k}}{\xi^{k}} \nabla_{x} \tilde{g}\left(x^{k}, \mu_{k}\right)+\sum_{j=1}^{m} \frac{\beta_{j}^{k}}{\xi^{k}} \nabla h_{j}\left(x^{k}\right)=0
$$

When $\mu_{k} \longrightarrow 0, x^{k} \longrightarrow x^{*}$; let $\left(\alpha^{k} / \xi^{k}, \beta_{j}^{k} / \xi^{k}\right) \longrightarrow$ $\left(\alpha^{*}, \beta_{j}^{*}\right.$, as $k \longrightarrow \infty$, and we have

$$
\begin{aligned}
& \lim _{k \rightarrow \infty}\left[\frac{\alpha^{k}}{\xi^{k}} \nabla_{x} \tilde{g}\left(x^{k}, \mu_{k}\right)+\sum_{j=1}^{m} \frac{\beta_{j}^{k}}{\xi^{k}} \nabla h_{j}\left(x^{k}\right)\right] \\
& \quad=\lim _{k \longrightarrow \infty}\left[\frac{\alpha^{k}}{\xi^{k}}\left(\mu_{k} \nabla \phi\left(x^{k}\right)+\left(\theta-\mu_{k}\right) \nabla_{x} g\left(x^{k}, \mu_{k}\right)\right)+\sum_{j=1}^{m} \frac{\beta_{j}^{k}}{\xi^{k}} \nabla h_{j}\left(x^{k}\right)\right] \\
& \quad=\lim _{k \longrightarrow \infty}\left[\frac{\alpha^{k}}{\xi^{k}}\left(\mu_{k} \nabla \phi\left(x^{k}\right)+\left(\theta-\mu_{k}\right) \sum_{i=1}^{m} \tau_{i}\left(x^{k}, \mu_{k}\right) \nabla g_{i}\left(x^{k}\right)\right)+\sum_{j=1}^{m} \frac{\beta_{j}^{k}}{\xi^{k}} \nabla h_{j}\left(x^{k}\right)\right] \\
& \quad=\alpha^{*} \theta \sum_{i=1}^{m} \tau_{i}\left(x^{*}, 0\right) \nabla g_{i}\left(x^{*}\right)+\sum_{j=1}^{m} \beta_{j}^{*} \nabla h_{j}\left(x^{*}\right)=0 .
\end{aligned}
$$

This is a contradiction with assumption (A2), so $\exists \theta_{3}>0$, for any $\mu \in\left(0, \theta_{3}\right]$, matrix $\left\{\left(\nabla_{x} \tilde{g}_{i}(x, \mu)_{\left.i \in I_{\mu}(x)\right\}}, \nabla h_{j}(x)\right\}\right.$ is positive linearly independent.
Define $\theta=\min \left\{\theta_{1}, \theta_{2}, \theta_{3}\right\}$. Since $\Omega_{\theta}^{0}$ is nonempty, for any $x^{0} \in \Omega_{\theta}^{0}, y^{0} \in R_{++}^{m}$, and $z^{0} \in R^{l}$, let $w^{0}=\left(x^{0}, y^{0}, z^{0}\right)$, and we construct the homotopy equation as follows:

$$
H\left(w, w^{0}, \mu\right)=\left(\begin{array}{c}
(1-\mu)\left[\nabla f(x)+\nabla_{x} \tilde{g}(x, \mu \theta) y+\nabla h(x) z\right]+\mu\left(x-x^{0}\right) \\
Y \tilde{g}(x, \mu \theta)+\mu \eta \\
h(x)-\mu z
\end{array}\right)=0,
$$

where $\quad w=(x, y, z) \in \Omega_{\mu} \times R_{+}^{m} \times R^{l}, \quad \eta \in R_{++}^{m}, \quad$ and $Y=\operatorname{diag}(y)$.

When $\mu=0$, homotopy equation (11) turns to the K-K-T system

$$
\left(\begin{array}{c}
\nabla f(x)+\theta \sum_{i=1}^{m} \nabla g_{i}(x) y_{i}+\sum_{j=1}^{l} \nabla h_{j}(x) z_{j} \\
Y \theta g(x) \\
h(x)
\end{array}\right)=0 .
$$

When $\mu=1$, homotopy equation (11), $H\left(w, w^{0}, 1\right)=0$, has a unique simple solution

$$
(x, y, z)=\left(x^{0}, y^{0}, z^{0}\right)=\left(x^{0},-\left[\operatorname{diag}\left(\tilde{g}\left(x^{0}, \theta\right)\right)\right]^{-1} \eta, h\left(x^{0}\right)\right) \text {. }
$$

The following lemmas from differential topology will be used in the next section. At first, let $U \subset R^{n}$ be an open set, and let $\phi: U \longrightarrow R^{p}$ be a $C^{\alpha}(\alpha>\max \{0, n-p\})$ mapping; we say that $y \in R^{p}$ is a regular value for $\phi$ if

$$
\text { Range }\left[\frac{\partial \phi(x)}{\partial x}\right]=R^{p}, \quad \forall x \in \phi^{-1}(y)
$$

Lemma 4 (see [31]). Let $V \subset R^{n}$ and $U \subset R^{m}$ be open sets, and let $\phi: V \times U \longrightarrow R^{k}$ be a $C^{\alpha}$ mapping, where $\alpha>\max \{0, m-k\}$. If $0 \in R^{k}$ is a regular value of $\phi$, then for almost all $a \in V, 0$ is a regular value of $\phi_{a}=F(a, \cdot)$.

Lemma 5 (see [31]). Let $\phi: U \subset R^{n} \longrightarrow R^{p}$ be $C^{\alpha}(\alpha>\max \{0, n-p\})$. If 0 is a regular value of $\phi$, then $\phi^{-1}(0)$ consists of some $(n-p)$-dimensional $C^{\alpha}$ manifolds.

Lemma 6 (see [31]). A one-dimensional smooth manifold is diffeomorphic to a unit circle or a unit interval.

\section{Main Results}

For a given $w^{0} \in \Omega_{\theta}^{0} \times R_{++}^{m} \times R^{l}$, rewrite $H\left(w, w^{0}, \mu\right)$ in homotopy equation (11) as

$$
H_{w^{0}}(w, \mu)=H\left(w, w^{0}, \mu\right) .
$$

The zero-point set of $H_{w^{0}}(w, \mu)$ is

$$
H_{w^{0}}^{-1}(0)=\left\{(w, \mu) \in \Omega_{\theta}^{0} \times R_{++}^{m} \times R^{l} \times(0,1]: H_{w^{0}}(w, \mu)=0\right\} .
$$


Lemma 7. If assumptions (A1) and (A2) hold, given $w^{0} \in \Omega\left\{\_\theta\right\} \widehat{0} \times R_{++}^{m} \times R^{l}$, and if there exists a smooth curve $\Gamma_{w^{0}}$ starting from $\left(w^{0}, 1\right)$ in $\Omega_{\mu \theta} \times R_{++}^{m} \times R^{l} \times(0,1]$, then it must be bounded.

Proof. If $\Gamma_{w^{0}} \subset \Omega_{\mu \theta} \times R_{++}^{m} \times R^{l} \times(0,1]$ is unbounded, there exists $\left(x^{k}, y^{k}, z^{k}, \mu_{k}\right) \subset \Gamma_{w^{0}}$, and $\left\|\left(x^{k}, y^{k}, z^{k}, \mu_{k}\right)\right\| \longrightarrow \infty$.

From the second equation of (11), we have

$$
Y^{k} \tilde{g}\left(x^{k}, \mu_{k} \theta\right)+\mu_{k} \eta=0 .
$$

By equation (17), $\quad Y^{k} \widetilde{g}\left(x^{k}, \mu_{k} \theta\right) \leq 0, i=1,2, \ldots$, $m, x^{k} \in \Omega_{\mu_{k} \theta}$, and $\mu_{k} \theta \leq \theta$, and by Lemma $2, \Omega_{\mu_{k} \theta}$ is also bounded, so $\left\{x^{k}\right\}$ is a bounded sequence. Therefore, $\left\{x^{k}\right\}$ must exist a convergent subsequence which is also denoted as $\left\{x^{k}\right\}$. Let $x^{k} \longrightarrow \hat{x}$ and $\left\|\left(y^{k}, z^{k}\right)\right\| \longrightarrow \infty$ as $k \longrightarrow \infty$. Denoting $I^{*}=\left\{i \in\{1,2, \ldots, m\} \mid y^{k} \longrightarrow \infty\right\}$, by (17), $I^{*} \subset I_{\widehat{\mu} \theta}(\widehat{x})$; therefore, we obtain

$$
\begin{aligned}
\tilde{g}_{i}\left(x^{k}, \mu_{k} \theta\right) & =-\mu_{k}\left(y_{i}^{k}\right)^{-1} \eta, \quad i \notin I^{*}, \\
\tilde{g}_{i}(\widehat{x}, \widehat{\mu} \theta) & =\lim _{k \longrightarrow \infty} \widetilde{g}_{i}\left(x^{k}, \mu_{k} \theta\right)=0, \quad i \in I^{*}, \text { i.e. } \hat{x} \in \partial \Omega_{\widehat{\mu} \theta} .
\end{aligned}
$$

From the first equation of (11), we have

$$
\left(1-\mu_{k}\right)\left(\nabla f\left(x^{k}\right)+\sum_{i=1}^{m} y_{i}^{k} \nabla_{x} \widetilde{g}_{i}\left(x^{k}, \mu_{k} \theta\right)+\sum_{j=1}^{m} \nabla h_{j}\left(x^{k}\right) z_{j}^{k}\right)+\mu_{k}\left(x^{k}-x^{0}\right)=0
$$

(i) When $\widehat{\mu}=1$, from the third equation of (11), we have $z^{k} \longrightarrow h(\hat{x})$ as $k \longrightarrow \infty$, which implies that $\left\{z^{k}\right\}$ is bounded. Hence, $\left\|y^{k}\right\|=\infty$ and $\hat{x} \in \Omega_{\theta}$. If $\|\left(1-\mu_{k}\right)$ $y_{k} \|<\infty$, without loss of generality, and suppose $\left(1-\mu_{k}\right) y^{k} \longrightarrow \hat{y}$, then $\hat{y}_{i}=0$ for $i \notin I_{\theta}(\hat{x})$ from the second equation of (11). Taking limits in (19), we have

$$
\begin{aligned}
x^{0} & =\widehat{x}+\lim _{k \rightarrow \infty}\left(1-\mu_{k}\right)\left(\nabla f\left(x^{k}\right)+\sum_{i=1}^{m} \nabla_{x} \tilde{g}_{i}\left(x^{k}, \mu_{k} \theta\right) y_{i}^{k}+\sum_{j=1}^{l} \nabla h_{j}\left(x^{k}\right) z_{j}^{k}\right) \\
& =\widehat{x}+\lim _{k \longrightarrow \infty} \sum_{i \in I_{\theta}(\hat{x})}\left(1-\mu_{k}\right) y_{i}^{k} \nabla_{x} \widetilde{\mathfrak{g}}_{i}\left(x^{k}, \mu_{k} \theta\right) \\
& =\hat{x}+\sum_{i \in I_{\theta}(\hat{x})} \widehat{y}_{i} \nabla_{x} \widetilde{\mathfrak{g}}_{i}(\hat{x}, \theta),
\end{aligned}
$$

but $\tilde{g}(x, \theta)=\theta \phi(x)$ is a convex function; this is impossible.

If $\left\|\left(1-\mu^{k}\right) y^{k}\right\| \longrightarrow \infty$, the discussion is the same as the following case (ii).

(ii) When $\widehat{\mu} \in(0,1)$, without loss of generality, suppose that $\left(1-\mu^{k}\right) y^{k} /\left\|\left(1-\mu^{k}\right) y^{k}\right\| \longrightarrow \widehat{\alpha}$ with $\|\widehat{\alpha}\|=1$ and $\widehat{\alpha}_{i}=0$ for $i \notin I_{\hat{\mu} \theta}(\widehat{x})$. Through dividing both sides of equation (19) by $\left\|\left(1-\mu^{k}\right) y^{k}\right\|$ and taking limits, we have

$$
\sum_{i \in I_{\hat{\mu} \theta}(\hat{x})} \widehat{\alpha}_{i} \nabla_{x} \tilde{g}_{i}(\widehat{x}, \widehat{\mu} \theta)=0
$$

which contradicts with Lemma 3. (iii) When $\widehat{\mu}=0$, without loss of generality, suppose that $\left(y^{k}, z^{k}\right) /\left\|\left(y^{k}, z^{k}\right)\right\| \longrightarrow(\widehat{\alpha}, t \widehat{\beta})$ with $\|(\widehat{\alpha}, t \widehat{\beta})\|=1$ and $\alpha_{i}=0$, for $i \notin I_{0}(\widehat{x})$. Through dividing both sides of equation (19) by $\left\|\left(y^{k}, z^{k}\right)\right\|$ and taking limits, we have

$$
\sum_{i \in I_{0}(\hat{x})} \widehat{\alpha}_{i} \nabla_{x} \widetilde{g}_{i}(\widehat{x}, 0)+\sum_{j=1}^{l} \beta_{j} \nabla h_{j}(\widehat{x})=0
$$

which contradicts with Lemma 3.

In conclusion, from the above discussion, we obtain that $\Gamma_{w^{0}}$ is a bounded curve in $\Omega_{\mu \theta} \times R_{++}^{m} \times R^{l} \times[0,1]$.

Theorem 1. Suppose assumptions (A1) and (A2) hold, for almost all $w^{0} \in \Omega_{\theta}^{0} \times R_{++}^{m} \times R^{l}$, the zero-point set of homotopy equation (11) contains a smooth curve $\Gamma_{w^{0}} \subset \Omega_{\theta} \times R_{+}^{m} \times R^{l} \times$ 
$(0,1]$ starting from $\left(x^{0}, y^{0}, z^{0}, 1\right)$, which terminates or approaches to the hyperplane $\mu \longrightarrow 0$. If $\left(x^{*}, y^{*}, z^{*}, 0\right)$ is a limit point of $\Gamma_{w^{0}}$, then $w^{*}=\left(x^{*}, y^{*}, z^{*}\right)$ is a solution to the $K-K-T$ system of problem (11).

Proof. Let $\quad \tilde{H}\left(w, x^{0}, \mu\right): \Omega_{\mu \theta} \times R_{+}^{m} \times R^{l} \times \Omega_{\theta}^{0} \times(0,1] \longrightarrow$ $\Omega_{\mu \theta} \times R_{+}^{m} \times R^{l}$ be the same map as $H\left(w, x^{0}, \mu\right)$ but taking $x^{0}$ as variate. Consider the following submatrix of the Jacobian $D H\left(w, x^{0}, \mu\right)$ :

$$
\frac{\partial \widetilde{H}\left(w, x^{0}, \mu\right)}{\partial\left(y, z, x^{0}\right)}=\left(\begin{array}{cccc}
* & * & -\mu I & \\
\operatorname{diag}(\widetilde{g} & (x, \mu \theta)) & 0 & 0 \\
0 & -\mu I & 0
\end{array}\right) .
$$

For all $\mu \in(0,1]$ and any $x^{0} \in \Omega_{\theta}^{0}$, from $(w, \mu) \in H_{w^{0}}^{-1}(0)$ and $Y \tilde{g}(x, \mu \theta)+\mu \eta=0$, we get that $\operatorname{diag}(\widetilde{g}(x, \mu \theta))$ is nonsingular, which implies that $\partial \tilde{H}\left(w, x^{0}, \mu\right) / \partial\left(y, z, x^{0}\right)$ is nonsingular.
Hence, matrix $D \tilde{H}\left(w, x^{0}, \mu\right)$ is full row rank. That is, 0 is a regular value of $\tilde{H}\left(w, x^{0}, \mu\right)$. By Lemma 4 , we have that, for almost all $x^{0} \in \Omega_{\theta}^{0}, 0$ is a regular value of $H\left(w, x^{0}, \mu\right)$.

Note that the matrix

$$
\frac{\partial H\left(w^{0}, w^{0}, 1\right)}{\partial w}=\left(\begin{array}{ccc}
I & 0 & 0 \\
* & \operatorname{diag}\left(\tilde{g}\left(x^{0}, \theta\right)\right) & 0 \\
* & 0 & -I
\end{array}\right)
$$

is nonsingular. From Lemma 5 , if 0 is a regular value of $H\left(w, w^{0}, \mu\right), \partial H\left(w^{0}, w^{0}, 1\right) / \partial w$ is nonsingular, and the fact $H\left(w^{0}, w^{0}, 1\right)=0, H_{w^{0}}^{-1}(0)$ must contain a smooth curve $\Gamma_{w^{0}}$ starting from $\left(x^{0}, y^{0}, z^{0}, 1\right)$ and going to $\Omega_{\mu \theta} \times R_{+}^{m} \times R^{l} \times[0,1]$. Then, from Lemma 6, the curve $\Gamma_{w^{0}} \subset \Omega_{\mu \theta} \times R_{+}^{m} \times R^{l} \times(0,1]$ must be diffeomorphic to a unit circle or a unit interval $[0,1)$.

We have that $\Gamma_{w^{0}}$ is not diffeomorphic to a unit circle. That is, $\Gamma_{w^{0}}$ is diffeomorphic to $[0,1)$. Let $\left(x^{*}, y^{*}, z^{*}, \mu_{*}\right)$ be a limit point of $\Gamma_{w^{0}}$; only the following five cases are possible:

$$
\begin{aligned}
& \text { (i) }\left(x^{*}, y^{*}, z^{*}\right) \in \Omega_{\theta} \times R_{+}^{m} \times R^{l} \mu_{*}=1\left\|\left(y^{*}, z^{*}\right)\right\|<\infty, \\
& \text { (ii) }\left(x^{*}, y^{*}, z^{*}\right) \in \Omega_{\mu_{*} \theta} \times R_{+}^{m} \times R^{l} \mu_{*} \in[0,1]\left\|\left(y^{*}, z^{*}\right)\right\|=\infty, \\
& \text { (iii) }\left(x^{*}, y^{*}, z^{*}\right) \in \Omega_{\mu_{*} \theta} \times \partial R_{+}^{m} \times R^{l} \mu_{*} \in(0,1)\left\|\left(y^{*}, z^{*}\right)\right\|<\infty, \\
& \text { (iv) }\left(x^{*}, y^{*}, z^{*}\right) \in \partial \Omega_{\mu_{*} \theta} \times R_{++}^{m} \times R^{l} \mu_{*} \in(0,1)\left\|\left(y^{*}, z^{*}\right)\right\|<\infty, \\
& \text { (v) }\left(x^{*}, y^{*}, z^{*}\right) \in \Omega \times R_{+}^{m} \times R^{l} \mu_{*}=0\left\|\left(y^{*}, z^{*}\right)\right\|<\infty .
\end{aligned}
$$

Since $\left(w^{0}, 1\right)$ is only one solution of the equation $H\left(w, w^{0}, 1\right)=0$ and $\left(\partial H\left(w^{0}, w^{0}, 1\right) / \partial w\right)$ is nonsingular, case (i) is impossible. From Lemma 7 , case (ii) is also impossible.

From $\operatorname{diag}\left(\tilde{g}\left(x^{*}, \mu_{*} \theta\right)\right) y^{*}+\mu_{*} \eta=0$, we have that $\mu_{*}>0$ and $y^{*} \in \partial R_{+}^{m}$, i.e., $y_{i}^{*}=0$, for some $1 \leq i \leq m$, cannot happen simultaneously. Therefore, case (iii) is impossible. If the multipliers $y^{*}>0$ and the homotopy parameter $\mu_{*}>0$, from $\operatorname{diag}\left(\widetilde{g}\left(x^{*}, \mu_{*} \theta\right)\right) y^{*}+\mu_{*} \eta=0$, we can $\operatorname{get} \operatorname{diag}\left(\widetilde{g}\left(x^{*}, \mu_{*} \theta\right)\right)<$ 0 , which implies that case (iv) is also impossible.

As a conclusion, case (v) is the only possible case. That is, curve $\Gamma_{w^{0}}$ must terminate in or approach to the hyperplane at $\mu_{*}=0$. And hence, $w^{*}=\left(x^{*}, y^{*}, z^{*}\right)$ is a solution to the $\mathrm{K}-\mathrm{K}-\mathrm{T}$ system of problem (1).

\section{Numerical Algorithm}

By Theorem 1, homotopy equation (11) generates a smooth curve $\Gamma_{w}^{0}$ for almost all $\left(w^{0}, \mu\right] \in \Omega_{\theta}^{0} \times R_{++}^{m} \times R^{l} \times(0,1)$ as $\mu \longrightarrow 0$, and one can find a solution of homotopy equation (11). Letting $s$ to be the arc length of $\Gamma_{w}^{0}$, we can parameterize $\Gamma_{w}^{0}$ with respect to $s$, i.e.,

$$
\begin{array}{r}
H(w(s), \mu(s))=0, \\
w(0)=w^{0}, \mu(0)=1 .
\end{array}
$$

By differentiating (26), we can get

$$
\begin{array}{r}
H^{\prime}(w(s), \mu(s))\left(\begin{array}{c}
\dot{w} \\
\dot{\mu}
\end{array}\right)=0, \\
\mu(0)=w^{0}, \mu(0)=1,
\end{array}
$$

where $H^{\prime}$ is the derivative of $H$.

As how to trace the homotopy path $\Gamma_{w^{0}}$ numerically, we can use the standard predictor-corrector procedure; for more details, see $[32,33]$. In this paper, our contribution is only the theoretical results about the proposed algorithm which only requires that any initial point can be chosen in the shifted feasible set but not necessarily in the original feasible set. The relative homotopy algorithms and numerical simulations on the performance for the proposed algorithm can be implemented as [34].

\section{Data Availability}

No data were used to support this study.

\section{Conflicts of Interest}

The authors declare no conflicts of interest. 


\section{Authors' Contributions}

Both authors contributed equally to this paper and read and approved the final manuscript.

\section{Acknowledgments}

Zhichuan Zhu was supported by the Education Department Foundation of Jilin Province (Grant no. JJKH20190742SK) and the Advanced Talents Research Fund of Liaoning University. Yeong-Cheng Liou was partially supported by MOST 109-2410-H-037-010 and MOST 108-2410-H-037020 and the Kaohsiung Medical University Research Foundation (Grant no. KMU-M108002).

\section{References}

[1] R. B. Kellogg, T. Y. Li, and J. Yorke, "A constructive proof of the brouwer fixed-point Theorem and computational results," SIAM Journal on Numerical Analysis, vol. 13, no. 4, pp. 473-483, 1976.

[2] S. Smale, "A convergent process of price adjustment and global Newton method," Journal of Mathematical Economics, vol. 3, pp. 1-14, 1976.

[3] S. N. Chow, J. Mallet-Paret, and J. A. Yorke, "Finding zeroes of maps: homotopy methods that are constructive with probability one," Mathematics of Computation, vol. 32, no. 143, p. $887,1978$.

[4] G. C. Feng and B. Yu, "Conbined homotopy interior point method for nonlinear programming problems," in Advances in Numerical Mathematics; Proceeding of the second JapanChina Seminar on Numerical Mathematics, Lecture Notes in Numerical and Applied Analysis, H. Fujita and M. Yamaguti, Eds., pp. 9-16, Kinokuniya, Tokyo, Japan, 1995.

[5] L.-C. Ceng, A. Petrusel, Y. Yao, and Y. Yao, "Hybrid viscosity extragradient method for systems of variational inequalities, fixed Points of nonexpansive mappings, zero points of accretive operators in Banach spaces," Fixed Point Theory, vol. 19, no. 2, pp. 487-502, 2018.

[6] B. Yu and Z. Lin, "Homotopy Methods for a class of nonconvex Brouwer fixed-point problems," Applied Mathematics and Computation, vol. 74, pp. 65-77, 1996.

[7] L. C. Ceng, A. Petrusel, J. C. Yao, and Y. Yao, "Systems of variational inequalities with hierarchical variational inequality constraints for Lipschitzian pseudocontractions," Fixed Point Theory, vol. 20, pp. 113-134, 2019.

[8] Z. Lin, B. Yu, and G. Feng, "A combined homotopy interior point method for convex nonlinear programming," Applied Mathematics and Computation, vol. 84, no. 2-3, pp. 193-211, 1997.

[9] G. Feng, Z. Lin, and B. Yu, "Existence of an interior pathway to a Karush-Kuhn-Tucker point of a nonconvex programming problem," Nonlinear Analysis: Theory, Methods \& Applications, vol. 32, no. 6, pp. 761-768, 1998.

[10] Y. Yao, L. Leng, M. Postolache, and X. Zheng, "Mann-type iteration method for solving the split common fixed point problem," Journal of Nonlinear and Convex Analysis, vol. 18, pp. 875-882, 2017.

[11] Z. Lin and Y. Li, "Homotopy method for solving variational inequalities," Journal of Optimization Theory and Applications, vol. 100, no. 1, pp. 207-218, 1999.

[12] Z. Lin, B. Yu, and D. Zhu, "A continuation method for solving fixed points of self-mappings in general nonconvex sets,"
Nonlinear Analysis: Theory, Methods \& Applications, vol. 52, no. 3, pp. 905-915, 2003.

[13] Y. Yao, M. Postolache, and J. C. Yao, "Iterative algorithms for generalized varational inequalities," University Politehnica of Bucharest Scientific Bulletin, vol. 81, pp. 3-16, 2019.

[14] Y. Yao, M. Postolache, and J. C. Yao, "Strong convergence of an extragradient algorithm for variational inequality and fixed point problems," UPB Scientific Bulletin, Series A: Applied, vol. 82, no. 1, pp. 3-12, 2020.

[15] Q. Xu and B. Yu, "Homotopy Method for nonconvex programming in unbounded set, Northeast," International Journal of Mathematics, vol. 21, pp. 25-31, 2005.

[16] Q. Xu, B. Yu, and G.-C. Feng, "Homotopy methods for solving variational inequalities in unbounded sets," Journal of Global Optimization, vol. 31, no. 1, pp. 121-131, 2005.

[17] Y. Yao, X. Qin, and J. C. Yao, "Projection methods for firmly type nonexpansive operators," Journal of Nonlinear and Convex Analysis, vol. 19, pp. 407-415, 2018.

[18] Y. Yao and N. Shahzad, "Strong convergence of a proximal point algorithm with general errors," Optimization Letters, vol. 6, no. 4, pp. 621-628, 2012.

[19] Q. Xu and B. Yu, "Solving the Karush-Kuhn-Tucker system of a nonconvex programming problem on an unbounded set," Nonlinear Analysis: Theory, Methods \& Applications, vol. 70, no. 2, pp. 757-763, 2009.

[20] X. Zhao, J. C. Yao, and Y. Yao, “A proximal algorithm for solving split monotone variational inclusions," UPB Scientific Bulletin, Series A: Applied, vol. 82, no. 3, pp. 43-52, 2020.

[21] B. Yu, G.-c. Feng, and S.-L. Zhang, "The aggregate constraint homotopy method for nonconvex nonlinear programming," Nonlinear Analysis: Theory, Methods \& Applications, vol. 45, no. 7, pp. 839-847, 2001.

[22] Z. Zhou, M. Su, Y. Shang, and F. Wang, "Global convergence analysis of the aggregate constraint homotopy method for nonlinear programming problems with both inequality and equality constraints," Optimization, vol. 67, no. 8, pp. 1247-1264, 2018.

[23] B. Yu and Y. Shang, "Boundary moving combined homotopy method for nonconvex nonlinear programming," Journal of Mathematical Research and Exposition, vol. 26, pp. 831-834, 2006.

[24] Y. Shang and B. Yu, "Boundary moving combined homotopy method for convex nonlinear programming and its convergence," Journal of Jilin University, vol. 44, pp. 357-361, 2006.

[25] L. Yang, B. Yu, and Q. Xu, "A combined homtopy infeasible interior point method for nonconvex programming," Pacific Journal of Optimization, vol. 8, pp. 89-101, 2012.

[26] S. Y. Cho, X. Qin, J. C. Yao, and Y. Yao, "Viscosity approximation splitting methods for monotone and nonexpansive operators in Hilbert spaces," Journal of Nonlinear and Convex Analysis, vol. 19, pp. 251-264, 2018.

[27] X. Wang, X. Jiang, and Q. Liu, "The constraint shifting combined homotopy method for bounded general programming problems," Journal of Information and Computing Science, vol. 8, pp. 1753-1762, 2010.

[28] H. Zegeye, N. Shahzad, and Y. Yao, "Minimum-norm solution of variational inequality and fixed point problem in Banach spaces," Optimization, vol. 64, no. 2, pp. 453-471, 2015.

[29] X. P. Zhao and Y. Yao, "Modified extragradient algorithms for solving monotone variational inequalities and fixed point problems," Optimization, vol. 69, pp. 1987-2002, 2020. 
[30] Y. Yao, M. Postolache, and J. C. Yao, “An iterative algorithm for solving the generalized variational inequalities and fixed points problems," Mathematics, vol. 7, 2019.

[31] G. L. Naber, Topological Method in Euclidean Space, Cambridge University Press, London, UK, 1980.

[32] E. L. Allgower and K. Georg, Introduction to Numerical Continuation Methods, SIAM Society for Industrial and Applied Mathematics, Philadelphia, PA, USA, 2003.

[33] J. Nocedal and S. J. Wright, Numerical Optimization, Springer, Berlin, Germany, Second edition, 2006.

[34] Z. Zhu and B. Yu, "A modified homotopy method for solving the principal-agent bilevel programming problem," Computational and Applied Mathematics, vol. 37, no. 1, pp. 541-566, 2018. 